\title{
Éditorial
}

\section{P. Lequien}

(C) Springer-Verlag France 2012

Ce fut une bonne idée de la Société française de médecine périnatale de décider en 2008 la création d'une revue rassemblant la production de tous ses acteurs, dans leur diversité. Cette idée était si bonne qu'on s'étonne aujourd'hui que près de quarante ans aient été nécessaires pour qu'elle s'impose. Il faut rendre hommage au Bureau de l'époque et à son président, Xavier Hernandorena, d'avoir décidé de se lancer dans cette aventure.

Ce fut une autre bonne idée et une chance d'établir un partenariat avec Springer-Verlag France. L'expérience de cet éditeur de dimension largement internationale a été précieuse, sinon indispensable. Les interlocutrices assignées à cette entreprise, Nathalie Huilleret, Méline Berthelot et Marie-Elia Come-Garry ont mis à la disposition de la Société non seulement leur savoir-faire et leur expérience, mais aussi une disponibilité sans faille.

Le choix du rédacteur en chef était-il une bonne idée ? Il n'appartient évidemment pas à l'auteur de ces lignes de répondre à cette question. Tout au plus peut-il proposer des explications à son recrutement. Il devait avoir une bonne, sinon une longue expérience de la Société et adhérer sans réserve à ses objectifs. Il devait être suffisamment affranchi des contraintes professionnelles quotidiennes pour consacrer le temps nécessaire à la concrétisation de ce projet : quoi de mieux qu'un « jeune » retraité ? Il devait avoir établi des liens à base de crédibilité et de sympathie avec les représentants des disciplines regroupées au sein de la Société. Il devait enfin, entre autres critères, être capable de réunir un conseil scientifique et surtout un comité de rédaction qui est en fait la structure opérationnelle de la
Revue. Le moment est venu de rendre hommage à ceux qui ont accepté d'emblée de s'investir dans cette tâche. Leur réunion bisannuelle est un moment de réflexion féconde dans le climat d'amitié préalable à toute collaboration de qualité. Il fut considéré comme une hardiesse d'y associer des représentants des « usagers » : cette décision n'a jamais été remise en cause. Bien au contraire, ces représentants ont répondu à l'attente. Ils ne sont pas les porte-paroles d'un quelconque lobby, d'une entreprise de dénigrement systématique. Leurs propos sont, bien sûr, et on serait tenté d'écrire " heureusement », parfois dérangeants en ce qu'ils remettent en cause notre conviction qu'il suffit de vouloir le bien des patients et des patientes pour le faire. En un temps où les progrès techniques marquent le pas, leur collaboration est indispensable au moment de la réflexion sur la qualité des soins.

C'est enfin une excellente idée, alors que le rédacteur en chef considère que le moment est venu de mettre un terme à son exercice, que d'avoir sollicité pour lui succéder le professeur Delphine Mitanchez, responsable du Service de néonatologie de l'hôpital Trousseau. Elle a accepté d'ajouter cette charge à toutes celles qu'elle a accumulées au cours des dernières années : son engagement mérite admiration et reconnaissance. S'agira-t-il d'une succession ou d'une rupture ? On pourrait être tenté de retenir le deuxième terme. Différence de sexe, de génération, et puis une parisienne en lieu et place d'un provincial : on pourrait allonger la liste des contrastes. C'est à elle qu'il appartiendra d'imprimer à la Revue, avec un Comité de rédaction élargi, une évolution souhaitable au bout de trois ans d'existence.

P. Lequien $(\varangle)$

81, boulevard Carnot, F-59420 Mouvaux, France

e-mail : pierre.lequien@aliceadsl.fr 